\title{
Two commonly used neoadjuvant chemoradiotherapy regimens for locally advanced stage III non-small cell lung carcinoma: Long-term results and associations with pathologic response
}

\author{
Mitchell Machtay, MDa \\ Jason H. Lee, MD ${ }^{\mathrm{b}}$ \\ James $P$. Stevenson, $M^{c}$ \\ Joseph B. Shrager, $M D^{d}$ \\ Kenneth M. Algazy, MD ${ }^{\mathrm{c}}$ \\ Joseph Treat, MD \\ Larry R. Kaiser, MD ${ }^{d}$
}

\begin{abstract}
From the Department of Radiation Oncology, University of Pennsylvania Medical Center, Philadelphia, $\mathrm{Pa},{ }^{\mathrm{a}}$ Hudner Oncology Center, Saint Anne's Hospital, Fall River, Mass, ${ }^{\text {b }}$ Department of Medical Oncology, University of Pennsylvania Medical Center, Philadelphia, $\mathrm{Pa}^{\mathrm{c}}{ }^{\mathrm{C}}$ Department of Thoracic Surgery, University of Pennsylvania Medical Center, Philadelphia, $\mathrm{Pa}^{\mathrm{d}}{ }^{\mathrm{d}}$ and Department of Medical Oncology, Fox Chase Cancer Center/Temple University, Philadelphia, $\mathrm{Pa}^{\mathrm{e}}$
\end{abstract}

Received for publication Feb 25, 2003; revisions requested March 27, 2003; revisions received June 30, 2003; accepted for publication July 16, 2003

Address for reprints: Mitchell Machtay, MD, Hospital of the University of Pennsylvania, Department of Radiation Oncology, 2 Donner Bldg, 3400 Spruce St, Philadelphia, PA 19104 (E-mail: machtay@xrt. upenn.edu).

J Thorac Cardiovasc Surg 2004;127:108-13 $0022-5223 / \$ 30.00$

Copyright ( $\odot 2004$ by The American Association for Thoracic Surgery

doi:10.1016/j.jtcvs.2003.07.027
Background: We performed this study to determine the outcomes (pathologic response, survival, local-regional control, and toxicity) in patients treated with neoadjuvant chemoradiotherapy and planned operation for stage IIIA non-small cell lung carcinoma.

Methods: Patients treated from 1993 to 2000 with neoadjuvant chemoradiotherapy and a predetermined plan for subsequent surgical resection for stage III non-small cell lung carcinoma were analyzed. All patients underwent pretreatment evaluation at the university's Multidisciplinary Lung Cancer Center. Most patients (87\%) had complete mediastinoscopy staging, and all were believed to be poor candidates for up-front operation because of bulky extent of disease. The radiotherapy program used conventional, 2-dimensionally planned treatment to 45 to $54 \mathrm{~Gy}$ in 1.8- to 2-Gy fraction size. Concurrent chemotherapy consisted of etoposide/cisplatin or carboplatin/paclitaxel. Study end points included resectability, pathologic response, localregional control, survival, and toxicity. An exploratory comparison between pathologic response and long-term survival was performed. An exploratory comparison between older chemotherapy (etoposide/cisplatin) and third-generation chemotherapy (carboplatin/paclitaxel) was also performed.

Results: Of 53 patients, 45 (85\%) were deemed surgical candidates after induction therapy. Twenty-two ( $42 \%$ of the initial cohort) patients had a major pathologic response to stage 0 , I, or II disease. The 5-year actuarial survival was $31 \%$. Major pathologic response was associated with improved survival $(48 \%$ vs $24 \% ; P=$ .027). The overall rate of early death potentially related to therapy in this series was 9\%; this mostly occurred in patients who underwent right pneumonectomy. There was no difference in efficacy or mortality between etoposide/cisplatin and radiotherapy versus carboplatin/paclitaxel and radiotherapy, although the latter regimen was associated with less grade 3 or higher acute toxicity necessitating interruption or hospitalization during neoadjuvant treatment $(P=.02)$. In-field local control was achieved in $83 \%$ of all patients (90\% of the patients who underwent resection). Brain metastases as the first site of treatment failure occurred in $23 \%$ of all patients.

Conclusions: Neoadjuvant concurrent chemoradiation delivers high resectability, major pathologic response rate, and excellent local-regional control, with encouraging long-term survival considering the patient population studied. Major pathologic response correlates with long-term survival. Neoadjuvant carboplatin/paclitaxel and radiotherapy is an appropriate framework on which to add new therapies. 


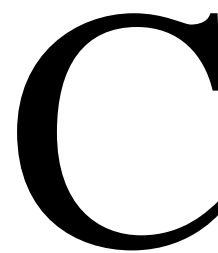

linical stage IIIA non-small cell lung carcinoma (NSCLC) is a major subgroup of locally advanced NSCLC. It is now the standard of care in the United States to incorporate systemic chemotherapy into the treatment of this disease. ${ }^{1,2}$ It is generally accepted that chemotherapy should be combined with local therapy consisting of radiotherapy (XRT), operation, or both. The Intergroup (INT) mechanism, led by the Radiation Therapy Oncology Group (RTOG), required 8 years to complete a large clinical trial (INT protocol 0139) comparing chemoradiation with or without operation-a difficult randomization for physicians and patients to accept. ${ }^{3}$ Preliminary results have just become available. ${ }^{4}$ In the meantime, institutions have performed smaller studies with a variety of forms of induction, or preoperative, therapy for clinical stage IIIA NSCLC. ${ }^{5-7}$

In the past decade, our institution has conducted a Multidisciplinary Lung Cancer Center, in which patients with complex thoracic oncologic problems are simultaneously evaluated by thoracic surgical, medical, and radiation oncologists. On the basis of a prospectively designed Center policy, our group has offered selected stage III NSCLC patients induction therapy before definitive operation. Those patients with clinically apparent $\mathrm{N} 2$ disease or other features that raised doubts about complete resectability were offered neoadjuvant chemoradiotherapy. We present the short-term and long-term outcomes (pathologic response, toxicity, local-regional control [LRC], and survival) from this clinical strategy.

\section{Patients and Methods}

This study analyzes the results of treatment at the University of Pennsylvania for clinical stage III NSCLC in which definitive operation was prospectively considered part of the treatment program. The time period extends from 1993 to 2000. Before 1993, patients with clinical stage III NSCLC were generally treated with radical XRT with or without chemotherapy (no operation). All patients in this series had good performance status (Zubrod scale $0-1)$ and no evidence of metastatic disease by computed tomographic (CT) scan of the chest/upper abdomen, bone scan, and head CT or magnetic resonance imaging. All patients underwent pulmonary function testing (at minimum, spirometry) before any treatment and were believed to have adequate pulmonary reserve to tolerate resection after neoadjuvant treatment.

All patients in this study were evaluated at the University of Pennsylvania Multidisciplinary Lung Cancer Center. Physician participants there include thoracic oncologic surgeons, medical oncologists, radiation oncologists, diagnostic radiologists, pathologists, and pulmonologists. In this series, all patients had locally advanced disease (stage III) that was judged to be marginally resectable on the basis of $\mathrm{CT}$ scan findings. Positive N2 nodes were those $1.5 \mathrm{~cm}$ or more in cross-sectional diameter. Histopathologic documentation of $\mathrm{N} 2$ disease by mediastinoscopy or other invasive procedure was strongly encouraged and was performed in 46 patients ( $87 \%$ of the patients in this series). However, some patients $(n=7)$ were offered neoadjuvant therapy solely on the basis of unequivocal imaging evidence of large $\mathrm{N} 2(\geq 4 \mathrm{~cm})$ nodes or clinical stage (by CT scan) T3-4 N1-2 disease because it was considered medically inappropriate to delay their treatment by several weeks to perform and allow healing from mediastinoscopy. Patients with N3 disease as determined by supraclavicular biopsy, CT scan, or mediastinoscopy were not offered this treatment program. Patients with T4 disease, as proven by thoracotomy, were also not offered this treatment, but selected patients with evidence of $\mathrm{T} 4$ disease by $\mathrm{CT}$ scan were included. To allow for a quality-assurance review of treatment delivery, for this study, only patients who had all of their treatment (chemotherapy, radiation therapy, and operation) at the University of Pennsylvania Medical Center were included.

\section{Treatment Regimens}

Briefly, the treatment program was as follows.

Radiotherapy. Radiation fields encompassed the primary tumor, ipsilateral hilum, and bilateral mediastinal nodal regions (minimum levels 2-7) to a dose of 45 to $54 \mathrm{~Gy}$, limiting the spinal cord dose to a maximum of $45 \mathrm{~Gy}$. Conventional 2-dimensional radiation planning was used, although computerized dosimetry was performed in all cases. Target volume definition was based on diagnostic and/or radiation treatment planning CT scans.

Chemotherapy. From 1993 to 1996, treatment consisted of neoadjuvant XRT with concurrent cisplatin/etoposide (EP). Systemic therapy consisted of 2 cycles of cisplatin $\left(60-100 \mathrm{mg} / \mathrm{m}^{2}\right)$ and etoposide $\left(120 \mathrm{mg} / \mathrm{m}^{2}\right.$ for 3 days) given during the first and fourth weeks of XRT, similar to the INT trial and the Southwest Oncology Group phase II multicenter study designs. ${ }^{8}$ Starting in 1997, the preferred chemotherapy regimen was gradually changed to concurrent carboplatin and paclitaxel on the basis of encouraging data presented in unresectable stage III NSCLC ${ }^{9}$ and the relative ease of outpatient administration with these drugs. Most of these more recent patients received weekly paclitaxel (45-50 mg/ $\mathrm{m}^{2}$ ) and a dose of carboplatin based on an area under the curve (AUC) formula (AUC = 2). Because of travel/logistic issues, several patients received 2 cycles of paclitaxel $\left(135 \mathrm{mg} / \mathrm{m}^{2}\right)$ and carboplatin $(\mathrm{AUC}=5)$ during the first and fourth weeks of XRT.

All patients underwent noninvasive re-staging (CT scans of the chest and head and bone scan) after induction therapy. Patients who maintained a high performance status and had no evidence of progressive disease underwent thoracotomy and attempted resection. Operation consisted of lobectomy (or pneumonectomy if necessary) with complete mediastinal lymph node dissection, as described by Mountain. ${ }^{10}$ In some cases, intercostal muscle flaps were used as additional protection for the bronchial stump; however, this was not routinely performed in all cases. There was no set institutional policy on postoperative or adjuvant therapy after surgery; only a few patients were given adjuvant chemotherapy or XRT after complete surgical resection. Details regarding adjuvant chemotherapy for these few patients and data on salvage chemotherapy for patients who experienced recurrence are, unfortunately, not available for this analysis.

\section{Outcome Analysis}

End points for this study included the following:

1. Resectability: proportion of patients who were able to undergo resection of tumor with no gross residual disease $\left(R_{0}\right.$ or $\mathrm{R}_{1}$ procedure). 
TABLE 1. Patient characteristics

\begin{tabular}{|c|c|c|c|}
\hline Characteristic & $\begin{array}{l}\text { Entire } \\
\text { cohort }\end{array}$ & $\begin{array}{l}\text { EP and XRT } \\
\text { group }\end{array}$ & $\begin{array}{c}\text { Carboplatin/ } \\
\text { paditaxel, } \\
\text { and XRT } \\
\text { group }\end{array}$ \\
\hline $\mathrm{n}$ & 53 & 22 & 31 \\
\hline Median age (y) & 62 & 63 & 61 \\
\hline Male sex & $29(55 \%)$ & $14(64 \%)$ & $15(48 \%)$ \\
\hline Clinical T3/T4 stage & $23(43 \%)$ & $14(62 \%)$ & $9(29 \%)$ \\
\hline Squamous cell histology & $10(21 \%)$ & $3(14 \%)$ & $7(27 \%)$ \\
\hline $\begin{array}{l}\text { Significant weight } \\
\text { loss }(>5 \%)\end{array}$ & $7(13 \%)$ & $2(9 \%)$ & $5(16 \%)$ \\
\hline Performance status 0 & $39(78 \%)$ & $20(91 \%)$ & $19(68 \%)$ \\
\hline $\begin{array}{l}\text { Median radiation dose } \\
\text { (Gy) }\end{array}$ & 48 & 46 & 50 \\
\hline
\end{tabular}

$E P$, Etoposide/cisplatin chemotherapy; $X R T$, radiotherapy.

2. Major pathologic response: proportion of patients who were resectable with negative margins, had marked histopathologic findings of tumor response, and were successfully downstaged to pathologic stage 0 , I, or II (T1-2 N0-1) disease.

3. LRC: freedom from clinically progressive intrathoracic or supraclavicular nodal disease, documented by biopsy where appropriate.

4. Overall survival: survival calculated from the date of diagnosis.

5. Severe toxicity: National Cancer Institute common toxicity criteria (version 2) grade 3 or greater treatment-related toxicity necessitating hospitalization.

Patients who had progressive disease and those who did not undergo resection for other reasons are still analyzed in this report on an intent-to-treat basis. Patients who did not proceed to operation because of early death, progression, or toxicity are still included in the survival analysis. Statistical analysis for differences in patient characteristics, resectability, pathologic staging, and toxicity was performed with a 2 -sided $\chi^{2}$ test. Survival was estimated actuarially by using Kaplan-Meier methods, ${ }^{11}$ and differences were assessed with the log-rank test. Statistical analysis was performed by one of the co-authors (J.H.L.) with Stata software (Stata Corp, College Station, Tex).

\section{Results}

A total of 53 patients were identified who satisfied the inclusion criteria. Twenty-two $(42 \%)$ received radiation with EP chemotherapy, and the other 31 patients (58\%) received carboplatin/paclitaxel chemotherapy. Characteristics of the patients are shown in Table 1 . There were no significant differences between the 2 treatment groups with respect to age, stage of disease, sex, or radiation dose. Patients who received EP chemotherapy presented with a higher proportion of clinical T3/T4 disease and more favorable performance status. Median follow-up was 57 months for all surviving patients, 65 months for patients who received EP chemotherapy, and 46 months for patients who received carboplatin/paclitaxel chemotherapy.

\section{Resectability and Major Response Rate}

After neoadjuvant therapy, operation was attempted for 45 $(85 \%)$ of the 53 initial patients. Obvious distant metastases developed in the other 8 patients, rendering operation inappropriate and thus making these patients inevaluable for assessment of local response. Thirty-eight of the 45 patients with operable disease had successful $\left(\mathrm{R}_{0}\right)$ complete resection of all gross tumor, for an overall resectability rate in this series of $72 \%$ (38/53). Three patients had an incomplete resection $\left(\mathrm{R}_{1}\right.$ or $\left.\mathrm{R}_{2}\right)$, and 4 patients were not operated on because of a lack of clinical response to induction therapy or death before surgery. A pathologic complete response was identified in 7 patients (16\% of operable patients, or $13 \%$ of the entire patient population). Pathologic stage I to IIA (T1-2 N0 M0 or T1 N1 M0) disease with marked histopathologic signs of a pathologic response to chemoradiation was found in an additional 15 patients. Thus, a major pathologic response occurred in $42 \%$ of the entire patient population (49\% of patients who underwent thoracotomy).

\section{Survival}

Median survival for all patients was 21 months. The 4- and 5 -year actuarial survivals were $31 \%$ for the entire patient population and $36 \%$ for the 45 patients who were considered operable and evaluable for response to neoadjuvant therapy. Patients treated with EP/XRT had a 4-year actuarial survival of $36 \%$, compared with $26 \%$ for carboplatin/paclitaxel and XRT. This was not a statistically significant difference $(P=.667)$. Initial performance status was also an important factor, because $40 \%$ of patients without significant cancer-related symptoms (performance status 0) were long-term survivors, whereas none of the performance status 1 patients survived beyond 3 years $(P=.015)$.

Patients who achieved a major pathologic response as a result of neoadjuvant therapy experienced more favorable survival compared with those who had less than a major response. The 4- and 5-year actuarial survival in the 22 patients with a major response was $48 \%$, compared with $24 \%$ among the 23 operable patients who did not gain a major response $(P=.027)$. Survival at 4 years was $0 \%$ for the 8 patients with progression. The survival curves based on response are shown in Figure 1. A summary of treatment outcomes is shown in Table 2.

Reanalysis of the survival data when excluding the 7 patients who did not have biopsy-proven $\mathrm{N} 2$ disease before neoadjuvant therapy does not change the results. The association between major response and long-term survival remains statistically significant $(P<.05)$.

\section{Patterns of Failure}

A total of 12 patients had local-regional relapses or progression, including 3 patients who underwent successful operation but later experienced isolated supraclavicular nodal 


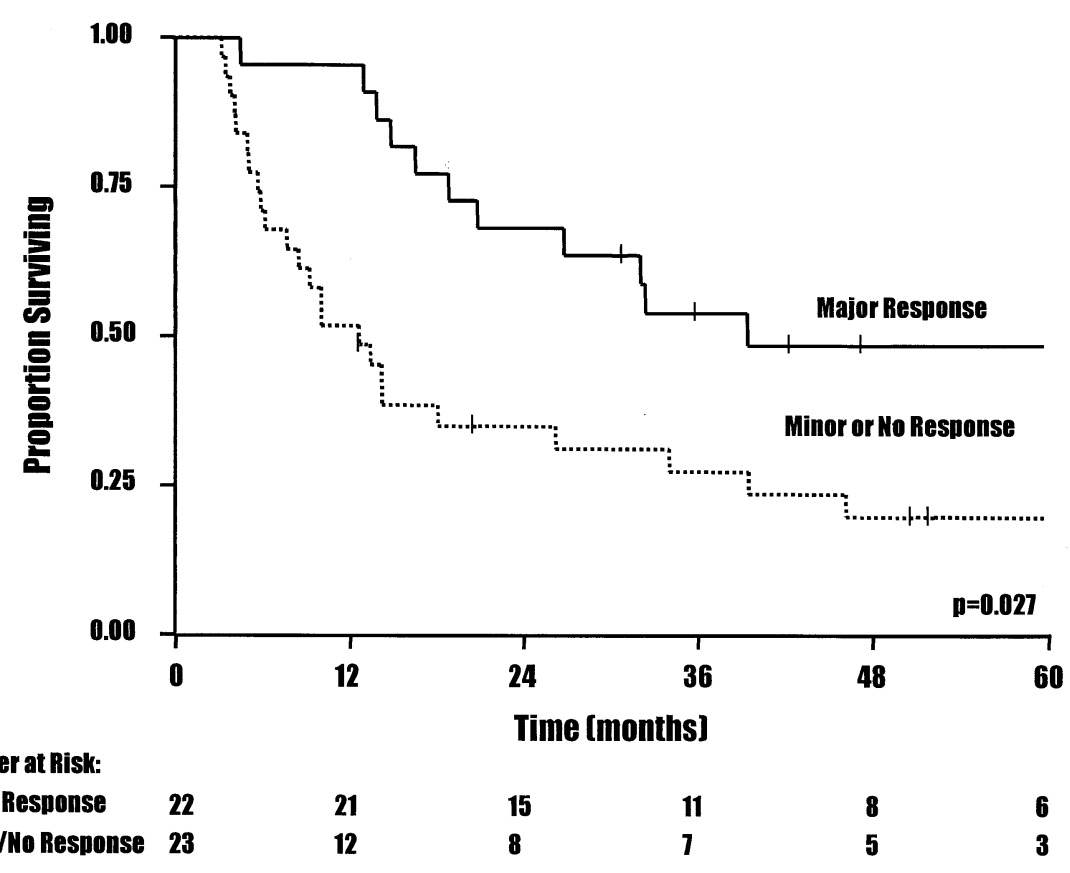

Figure 1. Overall survival as a function of response to neoadjuvant chemoradiotherapy.

failure outside of the radiation fields. The crude local control rate for the entire population was thus $77 \%$ (83\% local control within the radiation field). For the 41 patients who underwent operation, the crude local control rate (including the supraclavicular recurrences as local failures) was $83 \%$ (34/41). The local control rate within the surgical/radiotherapeutic fields for these 41 patients was 90\% (37/41). Among the 12 patients who did not undergo thoracotomy, 5 experienced local-regional progression, all within 1 year after XRT.

Brain metastases as the first site of failure occurred in 12 patients (23\%). Brain metastases occurred in 8 patients who underwent operation $(8 / 41 ; 19.5 \%)$ and 4 patients who did not undergo operation $(4 / 12 ; 33 \%)$. This is not statistically significant $(P=.31)$. There were no statistically significant differences in patterns of failure between EP/XRT and carboplatin/paclitaxel and XRT as neoadjuvant therapy.

\section{Morbidity and Mortality}

One patient died after induction chemoradiotherapy (before operation), and 2 patients died within 30 days after surgery, all from respiratory failure. Two additional patients died of respiratory disease between 30 and 90 days after surgery without definite evidence of recurrent cancer. The relationship between neoadjuvant therapy, operation, tumor, and intercurrent disease with these early deaths is uncertain. The overall rate of mortality potentially related to therapy in this series was $5(9 \%)$ of 53 . Three of these 5 patients had undergone right pneumonectomy. Early mortality occurred
TABLE 2. Outcome from treatment

\begin{tabular}{|c|c|c|c|}
\hline Outcome & $\begin{array}{l}\text { Entire } \\
\text { cohort }\end{array}$ & $\begin{array}{l}\text { EP and XRT } \\
\text { group }\end{array}$ & $\begin{array}{c}\text { Carboplatin/ } \\
\text { paditaxel } \\
\text { and XRT } \\
\text { group }\end{array}$ \\
\hline Severe acute toxicity & $7 / 53(13 \%)$ & $6 / 22(27 \%)$ & $1 / 31(3 \%)$ \\
\hline Progressive disease & $8 / 53(15 \%)$ & $2 / 22(9 \%)$ & $6 / 31(19 \%)$ \\
\hline Underwent resection & $41 / 53(77 \%)$ & 19/22 (86\%) & $22 / 31(71 \%)$ \\
\hline $\begin{array}{l}\text { Pathologic complete } \\
\text { response }^{*}\end{array}$ & $7 / 45(16 \%)$ & $3 / 20(15 \%)$ & $4 / 25(16 \%)$ \\
\hline $\begin{array}{l}\text { Major response } \\
\text { (stage } 0, \mathrm{I} \text {, or II)* }\end{array}$ & $22 / 45(49 \%)$ & $11 / 20(55 \%)$ & $9 / 25(44 \%)$ \\
\hline $\begin{array}{l}\text { Local-regional } \\
\text { control }\end{array}$ & $41 / 53(77 \%)$ & $17 / 22(77 \%)$ & $24 / 31(77 \%)$ \\
\hline Distant metastasis & $28 / 53(53 \%)$ & $13 / 22(59 \%)$ & $15 / 31(48 \%)$ \\
\hline $5-y$ survival & $31 \%$ & $36 \%$ & $26 \%$ \\
\hline
\end{tabular}

$E P$, Etoposide/cisplatin chemotherapy; $X R T$, radiotherapy.

*Proportion based on 45 patients without progressive disease during neoadjuvant therapy.

in 3 of 7 patients who underwent right pneumonectomy in this series. One of these deaths occurred 2 weeks after surgery from complications after a pulmonary embolism; 1 death resulted from a bronchopleural fistula and subsequent acute respiratory distress syndrome 10 weeks after surgery; and 1 death occurred from pneumonia at 4 weeks after surgery.

Temporary and reversible severe (grade 3) gastrointestinal toxicity (radiation esophagitis, nausea, or dehydration) developed in $7(13 \%)$ patients who underwent induction 
chemoradiotherapy, necessitating treatment interruption with or without a brief ( $<1$ week) hospitalization. This occurred in $6(27 \%)$ patients who received EP, compared with $1(3 \%)$ patient who received carboplatin/paclitaxel $(P$ $=.02)$.

\section{Discussion}

Our study shows that the resectability rates and LRC for stage IIIA NSCLC treated with neoadjuvant chemoradiotherapy are high. The survival data in this series are similar to phase II data reported by other studies of multimodality therapy for clinical stage III NSCLC, ${ }^{5,8}$ as well as the recently reported preliminary results of RTOG 93-09/INT 0139. ${ }^{4}$ It is disappointing that the overall survival and relapse-free survival have not improved appreciably over the past decade. This mainly reflects the inadequacy of current therapies to sterilize micrometastatic disease, including brain metastases.

This study included only patients with clinically obvious stage IIIA and highly selected stage IIIB disease who were not candidates for up-front operation. In this patient population, it is extremely controversial whether to use bimodality nonsurgical therapy (chemoradiation), bimodality surgical therapy (chemotherapy followed by operation), or trimodality therapy (chemoradiation followed by operation). RTOG 93-09/INT 0139 showed a progression-free survival but not an overall survival advantage to trimodality therapy compared with chemoradiation alone, and results are not fully matured. ${ }^{4}$ There is no published randomized trial comparing chemotherapy versus chemoradiotherapy as induction therapy for stage IIIA NSCLC; 2 small trials reported as meeting abstracts ${ }^{12,13}$ suggest better resectability and LRC (but not overall survival) with neoadjuvant chemoradiotherapy versus chemotherapy alone. The results of the large US INT trial (chemoradiation vs chemoradiation plus operation) and results from a large European trial (chemotherapy/surgery vs chemoradiation/surgery) will be extremely important. The US INT mechanism may open a randomized trial of this design in the next year.

One argument against the routine use of XRT as part of neoadjuvant regimens is that whereas published randomized trials show an advantage of neoadjuvant chemotherapy plus operation (with or without postoperative XRT) over operation alone (with or without postoperative XRT), ${ }^{14-16}$ there has not been an analogous trial to confirm that neoadjuvant chemoradiotherapy is superior to immediate operation. However, the results of randomized studies on neoadjuvant chemotherapy alone have been inconsistent. The 3 oftquoted trials referenced previously have very small sample sizes, and the long-term follow-up data are less impressive than the initial findings. ${ }^{17,18}$ A larger randomized study from France showed no survival benefit to neoadjuvant chemotherapy for stage IIIA NSCLC. ${ }^{19}$ These 3 studies may have had a population of patients with less bulky, less advanced disease than in our series or RTOG 93-09/INT 0139. A randomized study from the Lung Cancer Study Group showed no advantage to preoperative chemotherapy over preoperative XRT. ${ }^{20}$ A small randomized trial from the National Cancer Institute of Canada showed no difference between chemotherapy followed by operation versus XRT alone for stage IIIA NSCLC. ${ }^{21}$ Again, it should be emphasized that the conflicting results from all of these trials may indicate substantial differences in patient entry characteristics, such as the extent of IIIA disease (minimal volume vs bulky).

A second argument against the use of XRT as part of neoadjuvant regimens is that it obfuscates the ability to use pathologic response as a potential marker of long-term prognosis. Our study suggests that pathologic response is a useful predictor for long-term survival after chemoradiation. Pathologic responders had improved LRC and overall survival compared with nonresponders. Our data suggest that pathologic response is probably a viable early surrogate end point in future trials of marginally resectable NSCLC treated with XRT as a component of neoadjuvant therapy.

Although achieving successful resection and LRC does not guarantee long-term survival, it seems to be a necessary component of treatment success. Only 1 of 12 patients who did not undergo resection in our series was alive beyond 3 years of follow-up, and 5 patients had local-regional progression. Given the morbidity of local-regional failure, we contend that LRC is an important end point to consider in treatment decision-making for NSCLC.

Our study shows that serious toxicity was relatively high, although acceptable. Treatment-related mortality was not noticeably worse than with radical operation alone ${ }^{22}$ or chemotherapy followed by operation. ${ }^{23}$ Because XRT doses are limited to 45 to $54 \mathrm{~Gy}$, the risk and duration of grade 3 esophagitis is lower than that with definitive chemoradiation alone. Esophagitis seemed to be further reduced by the switch from high-dose EP to carboplatin/paclitaxel, with no loss of antitumor efficacy. It was disappointing to note that the change from EP to carboplatin/paclitaxel did not measurably improve outcomes; however, this is not surprising in light of data from large stage IV NSCLC chemotherapy studies. $^{24}$

Our study is inherently limited by its retrospective, nonrandomized design and has potential for significant patient selection bias. However, this study, although not randomized, does represent consecutive patients cared for by the same team of oncologists according to preset institutional treatment policies. As indicated by Table 1, the patient population cannot be considered a favorable subgroup of IIIA NSCLC. Until conclusive results from large randomized studies are available, institutional series such as ours provide hypothesis-generating data that are relevant for 
future trial development and clinical practice. Future prospective trials in our institution will build on a foundation of neoadjuvant paclitaxel/carboplatin and XRT combinations by judiciously adding targeted therapies.

\section{References}

1. Clinical practice guidelines for the treatment of unresectable nonsmall-cell lung cancer. Adopted on May 16, 1997 by the American Society of Clinical Oncology. J Clin Oncol. 1997;15:2996-3018.

2. Stewart LA. Chemotherapy in non-small cell lung cancer: a metaanalysis using updated data on individual patients from 52 randomised clinical trials. Non-small Cell Lung Cancer Collaborative Group. BMJ. 1995;311:899-909.

3. Byhardt RW. The evolution of Radiation Therapy Oncology Group (RTOG) protocols for nonsmall cell lung cancer. Int J Radiat Oncol Biol Phys. 1995;32:1513-25.

4. Albain KS, Scott CB, Rusch VR, Turrisi AT, et al. Phase III comparison of concurrent chemotherapy plus radiotherapy (CT/RT) and $\mathrm{CT} / \mathrm{RT}$ followed by surgical resection for stage IIIA (pN2) non-small cell lung cancer (NSCLC): initial results from intergroup trial 0139 (RTOG 93-09) [abstract 2497]. Proc Am Soc Clin Oncol. 2003;22:621.

5. Eberhardt W, Wilke H, Stamatis G, Stuschke M, Harstrick A, Menker $\mathrm{H}$, et al. Preoperative chemotherapy followed by concurrent chemoradiation therapy based on hyperfractionated accelerated radiotherapy and definitive surgery in locally advanced non-small cell lung cancer: mature results of a phase II trial. J Clin Oncol. 1998;16:622-34.

6. Greco FA, Hainsworth JD. Multidisciplinary approach to potentially curable non-small cell carcinoma of the lung. Oncology. 1997;11:2736.

7. Martini N, Kris MG, Gralla RJ, et al. The effects of preoperative chemotherapy on the resectability of non-small cell lung carcinoma with mediastinal lymph node metastases (N2M0). Ann Thorac Surg. 1988;45:370-9.

8. Albain KS, Rusch VW, Crowley JJ, Rice TW, Turrisi AT, Weick JK, et al. Concurrent cisplatin/etoposide plus chest radiotherapy followed by surgery for stages IIIA (N2) and IIIB non-small cell lung cancer: mature results of Southwest Oncology Group phase II study 8805. J Clin Oncol. 1995;13:1880-92.

9. Belani CP, Aisner J, Day R, et al. Weekly paclitaxel and carboplatin with simultaneous thoracic radiotherapy for locally advanced nonsmall cell lung cancer: three year followup [abstract 1608]. Proc Am Soc Clin Oncol. 1997;12:448a.

10. Mountain CF. Surgery for stage IIIa-N2 non-small cell lung cancer. Cancer. 1994;73:2589-98.

11. Kaplan EL, Meier P. Nonparametric estimation from incomplete observations. J Am Stat Assoc. 1958;53:457-81.
12. Fleck J, Camarzo J, Godoy D, et al. Chemoradiation therapy versus chemotherapy alone as neoadjuvant treatment for stage III non-small cell lung cancer: preliminary report of a phase III prospective randomized trial [abstract]. Proc Am Soc Clin Oncol. 1993;12:333.

13. Sauvaget J, Rebischung J, Vannetzel J. Phase III study of neoadjuvant MVP versus MVP plus chemoradiotherapy in stage III NSCLC [abstract 1935]. Proc Am Soc Clin Oncol. 2000;19:495a.

14. Rosell R, Gomez-Codina J, Camps C, et al. A randomized trial comparing preoperative chemotherapy plus surgery with surgery alone in patients with non-small cell lung cancer. $N$ Engl J Med. 1994;330: 153-8.

15. Roth JA, Fossella F, Komaki R, et al. A randomized trial comparing perioperative chemotherapy and surgery with surgery alone in resectable stage IIIA non-small cell lung cancer. J Natl Cancer Inst. 1994; 86:673-80

16. Pass HI, Pogrebniak HW, Steinberg SM, Mulshine J, Minna J. Randomized trial of neoadjuvant therapy for lung cancer: interim analysis. Ann Thorac Surg. 1992;53:992-8.

17. Rosell R, Gomez-Codina J, Camps C, et al. Preresectional chemotherapy in stage IIIA non-small cell lung cancer: a 7-year assessment of a randomized controlled trial. Lung Cancer. 1999;26:7-14.

18. Roth JA, Atkinson EN, Fossella F, Komaki R, Bernadette-Ryan M, Putnam JB Jr, et al. Long-term follow-up of patients enrolled in a randomized trial comparing perioperative chemotherapy and surgery with surgery alone in resectable stage IIIA non-small-cell lung cancer. Lung Cancer. 1998;21:1-6.

19. Depierre A, Milleron B, Moro-Sibilot D, et al. Preoperative chemotherapy followed by surgery compared with primary surgery in resectable stage I (except T1N0), II, and IIIA NSCLC. J Clin Oncol. 2002;20:247-53.

20. Wagner H, Lad T, Piantadosi S, Ruckdeschel JC. Randomized phase 2 evaluation preoperative radiation therapy and preoperative chemotherapy with mitomycin, vinblastine, and cisplatin in patients with technically unresectable stage IIIA and IIIB non-small cell cancer of the lung: LCSG 881. Chest. 1994;106:348S-354S.

21. Shepherd FA, Johnston MR, Payne D, et al. Randomized study of chemotherapy and surgery versus radiotherapy for stage IIIA nonsmall cell lung cancer: a National Cancer Institute of Canada Clinical Trials Group Study. Br J Cancer. 1998;78:683-5.

22. Ginsberg RJ, Hill LD, Eagan RT, et al. Modern thirty-day operative mortality for surgical resections in lung cancer. $J$ Thorac Cardiovasc Surg. 1983;86:654-8.

23. Roberts JR, DeVore RF, Carbone DP, et al. Neoadjuvant chemotherapy increases perioperative complications in patients undergoing resection for NSCLC [abstract 1794]. Proc Am Soc Clin Oncol. 1999;18.

24. Schiller JH, Harrington D, Belani CP, et al. Comparison of four chemotherapy regimens for advanced NSCLC. $N$ Engl J Med. 2002; 346:92-8. 